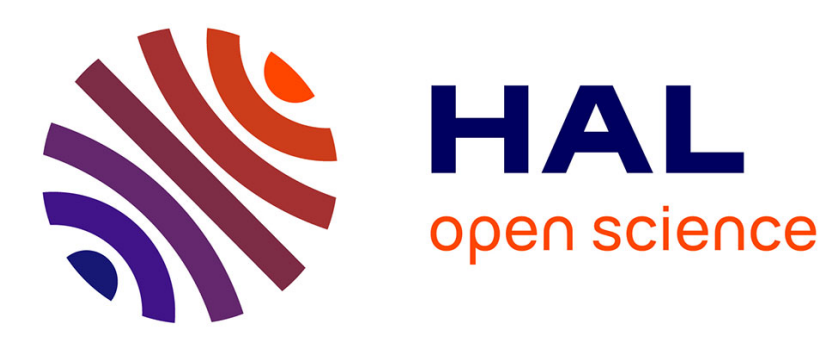

\title{
Visible Light Inter-Vehicle Communication for Platooning of Autonomous Vehicles
}

Mohammad Abualhoul, Oyunchimeg Shagdar, Fawzi Nashashibi

\section{To cite this version:}

Mohammad Abualhoul, Oyunchimeg Shagdar, Fawzi Nashashibi. Visible Light Inter-Vehicle Communication for Platooning of Autonomous Vehicles. 2016 IEEE Intelligent Vehicles Symposium IV2016, Jun 2016, Gothenburg, Sweden. hal-01308430

\section{HAL Id: hal-01308430 \\ https://hal.inria.fr/hal-01308430}

Submitted on 27 Apr 2016

HAL is a multi-disciplinary open access archive for the deposit and dissemination of scientific research documents, whether they are published or not. The documents may come from teaching and research institutions in France or abroad, or from public or private research centers.
L'archive ouverte pluridisciplinaire HAL, est destinée au dépôt et à la diffusion de documents scientifiques de niveau recherche, publiés ou non, émanant des établissements d'enseignement et de recherche français ou étrangers, des laboratoires publics ou privés. 


\title{
Visible Light Inter-Vehicle Communication for Platooning of Autonomous Vehicles
}

\author{
Mohammad Y. Abualhoul ${ }^{\dagger}$, Oyunchimeg Shagdar ${ }^{\dagger *}$ and Fawzi Nashashibi ${ }^{\dagger}$ \\ RITS Team - INRIA Paris Rocquencourt B.P. 105, 78153 Le Chesnay, France ${ }^{\dagger}$ \\ VEDECOM Institute, 77, rue des Chantiers, 78000 Versailles, France* \\ \{mohammad.abu_alhoul, oyunchimeg.shagdar, fawzi.nashashibi\}@inria.fr
}

\begin{abstract}
In this paper, we study a use of Visible Light Communication (VLC) technology for a platoon of autonomous vehicles. We present a low-cost, low-latency and simple outdoor VLC prototype, which can be installed as a vehicular taillighting system. The architecture of our VLC system is introduced, followed by performance evaluation with an especial attention on the VLC link resilience to ambient noise and communication range. Through the experiments, we observe that a use of proper optical filter stage at the receiver side, together with narrowing the transmitter Field-of-view (FOV), result in an extended communication range and make the VLC system more resilient to the ambient noises. Experimental results show that the system can provide 30 meters of intervehicle communication with $36 \mathrm{~ms}$ of latency, yet on sunny day conditions. The benefit of using the VLC system for platooning control is showed using a Simulink system that integrates our VLC platform for inter-communications to simulates the performance of autonomous vehicles platoon.
\end{abstract}

Keywords: VLC, Platoon, ITS, Road safety applications.

\section{INTRODUCTION}

Road safety applications of Intelligent Transportation Systems (ITS) require high communication reliability and low latency. Although the IEEE802.11p technology [1] is standardized to facilitate the V2X communication for such applications, a large number of studies reported that this technology still experiences some drawbacks, such as the channel congestion and the increased delay in highly dense vehicular traffic scenarios [2]. In recent years, Visible Light Communication (VLC) is emerging as a secure and costeffective technology for data transmission with a broad range of potential applications. In this work, we focus on testing the use of VLC as an integral supportive technology for ITS road safety applications, such as platooning. In this application, a specific number of vehicles form a group and travel together at high speed with relatively small inter-vehicle distances.

Indeed, one of the most efficient ways to increase road capacity and improve safety in highly dense vehicular traffic scenarios; is by driving vehicles in platoons with constant inter-vehicle spaces [3]. According to [4] and [5], road capacity can be increased dramatically by the use of tightly spaced platoon formation as expressed by the following formula:

$$
C=v \frac{n}{n l+(n-1) D+d},
$$

where $d$ represents the inter-platoon distance, $D$ the intraplatoon distance, $l$ the vehicle length, $v$ the steady-state speed, and $n$ the number of vehicles in each platoon.
As the constant spacing model suggest, relatively high dense vehicular scenarios can be managed efficiently using platoon formations. Although using radio technology for intra-platoon communication have been previously studied [6],[7], the improvement of the traffic capacity can on the other hand dramatically increase channel congestion and delay of the radio channel. Maintaining safety requirements for road applications with the presence of this trade-off between road capacity and channel congestion was the main motivation behind suggesting VLC as Vehicle-to-Vehicle (V2V) supportive communication link between platoon members. The use of vehicular tail-lighting to exchange information between platoon members as depicted in Fig 1. can play a key role in reducing the channel congestion.

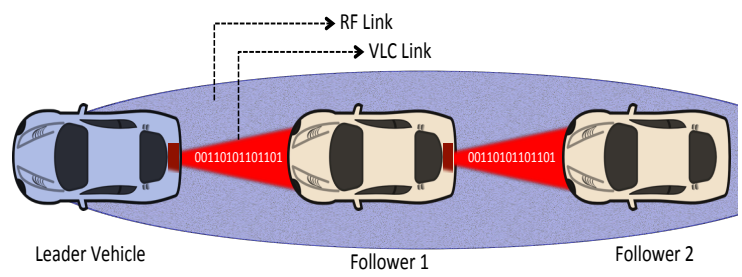

Fig. 1. VLC used for intra-platoon communications.

VLC technology showing significant progress in last decades by taking advantage of several accumulated knowledge and resources such as infrared [8] and the recent advances on high power Light Emitting Diodes (LEDs). Nowadays, LED takes place in every modern lighting system including vehicular lighting and signaling systems [9], the low cost and high switching speed expected to provide an opportunistic deployment of VLC in the short run.

Also, coast-efficiency and security are few of many other interesting proprieties make VLC more suitable candidate to be deployed as supportive communication technology for the proposed application in this work. While many existing efforts have investigated how VLC can be applied to vehicular applications, very few studies have analyzed and demonstrated the technology in real world conditions [10].

In this paper, we extend our previous study [12] for the two key elements necessary to recognize VLC technology in ITS applications: (i) the feasibility of VLC for outdoor conditions and under constraints posed mainly by the ambient noise and day light conditions, (ii) the capability of this technology to 
satisfy the vehicular platooning application safety requirements. This work makes the following contributions:

- We develop a VLC prototype by taking into account of the followings. 1) Using precise optical filtering techniques at the receiver end, allowing us to increase the prototype robustness against ambient noise. 2) Using off-the-shelf components in our implementation, with the observance of the standard feasible form factor of the vehicular lighting system.

- We evaluate the performance of the VLC prototype under daylight conditions and for Line-of-sight (LOS) scenario.

- The performance of platooning control, which uses our VLC platform for inter-vehicle communication is evaluated using a Simulink model.

The remainder of this paper is organized as follows. Section $\Pi$ identify the requirements for the platoon as hardsafety application. Section III introduces our VLC system architecture with the details of the prototype transmitter and receiver. In section IV, we report the experimental setup, together with the experimental results. Finally, simulation evaluation of VLC-based platoon using Simulink model is presented in $\mathrm{V}$ Conclusion and future work are given in Section VI

\section{Platooning APPLICATION REQUIREMENTS}

The standardized vehicular communications technology specifies the vehicle safety services and their performance requirements based on two primary metrics. i) Packet-DeliveryRatio (PDR), which indicate the ratio between the number of the successfully received packets and the number of transmitted packets. ii) Latency, the time needed of a packet to reach its destination.

TABLE I

SAFETy APPlications MaXimum Latency

\begin{tabular}{lll}
\hline Message Type & Use cases & Latency \\
\hline State Monitor & road condition & 100 to $1000 \mathrm{~ms}$ \\
Control & Cooperative driving & $100 \mathrm{~ms}$ \\
Infotainment & news, media & second level \\
Warning & lane-change, over-taking, collision & 10 to $100 \mathrm{~ms}$ \\
\hline
\end{tabular}

According to the US department of transportation, 8 out of 75 ITS services were recognized as a hard safety with respect to the safety applications requirements [13]. As detailed in TABLEI, the highest priority safety services such as crash avoidance and lane change warning target $100 \%$ PDR level and latency of 10 to $100 \mathrm{~ms}$ at the utmost. In our study, we consider these hard safety requirements for the VLC-based inter-vehicle communication for platoons.

\section{ARChitecture of VLC Platform}

The implementation of VLC platform proposes to exploit the vehicular tail-lights system and reduce both cost and installation complexity. In order to realize the VLC double functionality of both data transmission and vehicular lighting, the transmitter wavelength chosen to be in red color visible spectrum range $(635 \mathrm{~nm})$ and an emission power of 3 Watt for each of the 6 LED array, representing the vehicle taillights. As for the receiver end, the main components are the silicon Photo-Diode (PD) with a switchable gain detector PDA100A, and the optical band-pass filter with an operation band match the LED emission wavelength. Fig 2 shows the VLC overall transmitter and receiver block diagrams. As illustrated in the figure, in adding to the VLC ends, the transmitter and receiver architectures consist RTMaps platforms and Arduino micro-controllers. RTMAps is used to prepare specific packet format and connect the transmitter and receiver component with the micro-controller.

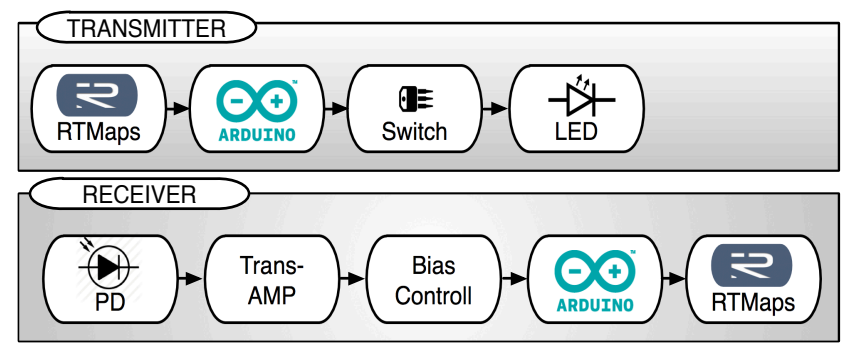

Fig. 2. The transmitter and receiver diagrams of the VLC platform.

For processing simplicity, a low-cost Mini-pro Arduino boards based on ATmega328P micro-controller has been used at both transmitter and receiver ends to perform modulation/demodulation, line coding, signal processing and interfacing the vehicles controller area network (CAN-BUS).

\section{A. VLC Transmitter}

The transmitter unit is composed of three main components, LEDs with mounted focusing lenses on the top of each, driving circuit and Arduino micro-controller as depicted in Fig 4 (a). In this implementation, 6 high-power (3Watt) LEDs illuminate $635 \mathrm{~nm}$ red-light data beam in which calibrated to represent the vehicle tail-light intensity.

The vehicle CAN-BUS data are packed into a specific packet format, where RTMaps [14] extracts the vehicle status (speed, acceleration and steering angle) accompanied with timestamp and packet counter, then generating a particular packet format as shown in Fig 3 These data are then adapt Manchester line coding in the micro-controller before modulating the LED array.

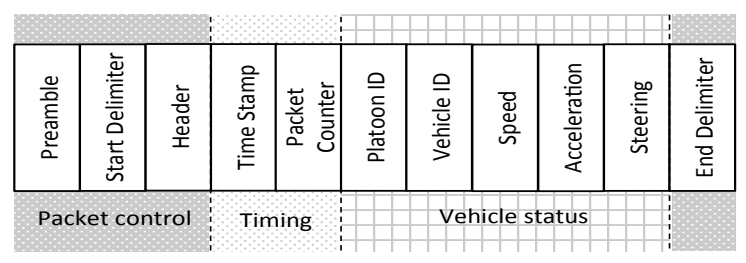

Fig. 3. Packet format for intra-platoon communication.

Manchester coding (also known as phase encoding) was used here based on the fact that the energy component of the encoded signal is not dependent on the data itself. Also, each transmitted bit in this context contains the same amount of energy and results in a constant intensity over transmission, 
either we send 0's or 1's, which ensure that human eye will never observe any light intensity fluctuation during data transmission.

The transmitter LED's array is then driven by the coded data using high-speed MOSEFET switching circuit, which applies the On-Off-Keying (OOK) intensity modulation.

\section{B. VLC Receiver}

The receiver unit depicted in Fig 4 (b \& c) composed of four main components: an optical filter, photodiode, tansimpedance amplifier and decision circuit. The optical filter was chosen specifically to operate in a narrow bandwidth $(635 \mathrm{~nm}+/-1 \mathrm{~nm})$ in order to provide immunity against any ambient noise source outside this filter range, and at the same time match the transmitter LED emission wavelength.

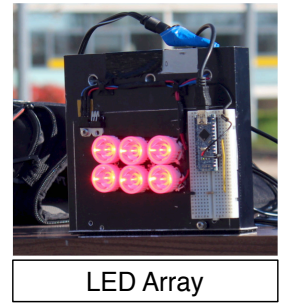

(a)

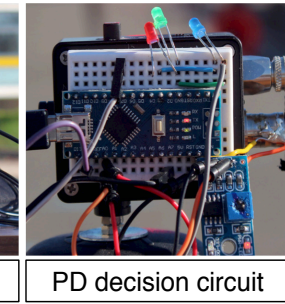

(b)

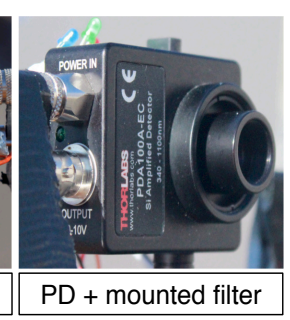

(c)
Fig. 4. VLC transmitter and receiver ends.

The used decision circuit LT1715 has signal threshold voltage comparator with a reference voltage $2.1 \mathrm{~V}$. This stage represents the receiver sensitivity, where the comparison reference value has been obtained empirically based on the impact of the ambient noise levels for many different scenarios and different receiver/transmitter positioning. Here, we adopt the simple Intensity Direct Detection (IDD) method.

\section{Channel model}

VLC channel characteristics are defined mainly by the LED lambertian emission pattern and both transmitter and receiver physical characteristics [8], where the path-loss can be presented as:

$$
P_{\text {loss }}=\left\{\begin{array}{cc}
\frac{(m+1) A_{p h}}{2 \pi d^{n}} \cos ^{m}(\varphi)(\psi) \cos (\psi), & 0<\psi<\psi_{c} \\
0 & \text { elsewhere }
\end{array}\right.
$$

For VLC path-loss theoretical model, the path-loss exponent $n$ is equal to 2 , where $d$ is the separation distance between transmitter and receiver, $\psi_{c}$ is the PD field of view (FOV) representing the maximum incidence angle. $A_{p h}$ is the physical area of the PD and $m=-\frac{\ln 2}{\ln (\cos \hat{\phi})}$ is lampertian emission order, which is a key parameter specifying the directivity of the transmitter. The LED half-power angle $\hat{\phi}$ has a remarkable influence on the coverage range and pattern shape of the lambertian light source. By choosing narrower $\hat{\phi}$, we can directly increase the communication range range.

\section{EXPERIMENTAL SETUP AND RESULTS}

In our previous work [12], [15], we have investigated the theoretical VLC channel model and evaluated its feasibility for platoon application on simulation basis, the two studies addressed the technology limitations and proposed a solution to enhance the FOV for sharp curvature scenarios. In this work, we step forward and compare the existing model with empirical results obtained experimentally from our developed platform.

\section{A. Experimental setup}

Figure 5 shows the outdoor VLC experimental setup, this setup was dedicated to studying the range and the communication link quality in outdoor environment and for a direct LOS, where more sophisticated scenarios such as maneuvers and Lane-change are intended to be our ongoing work. All tests were performed in a sunny daytime of December the 12th between 12:00 and 14:00, Paris time. Multiple iterations of data recording were performed at the receiver end, in which we record data for each $+500 \mathrm{~cm}$ increment in $d$, starting from $d=1$ meter.

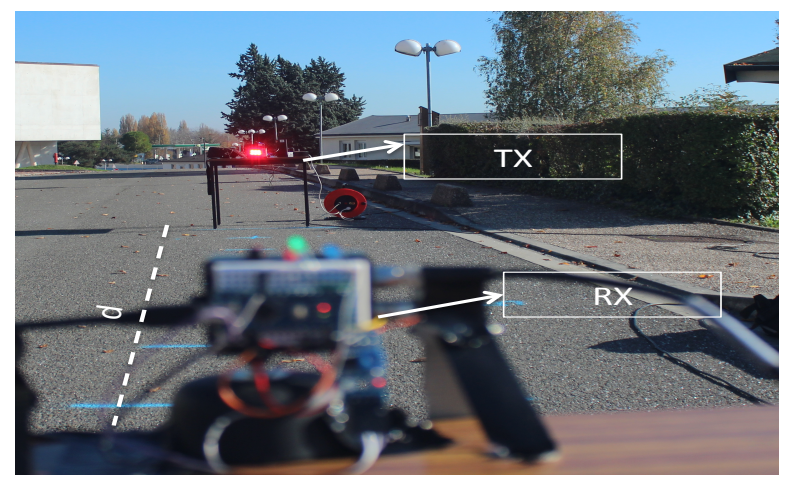

Fig. 5. Experimental setup

An independent power source supplied the transmitter module with constant 24 voltage and $700 \mathrm{~mA}$ in order to bias the LED array. Receiver module was also supplied by an independent power source in order to enable the trans-impedance amplifier. Arduino micro-controllers at both transmitter and receiver ends were powered by the 5 Volt USB connection and the height were fixed to $85 \mathrm{~cm}$ from the ground level. More parameters regarding the experimental setup are given in TABLE II.

\section{B. Experimental results}

In our LOS scenario, the distance between transmitter and receiver ends is much greater than optical detector size $A_{p h}$. Moreover, the terms $\cos ^{m}(\varphi)$ and $\cos (\theta)$ can set to equal 1 by maintaining aligned direct distance between both transmitter and receiver, where $\theta=\varphi=0$. It thus follows that one can simplify the path-loss expression in (2) and write the received power with respect to the transmission power as:

$$
P_{R}=P_{L o s s} P_{T}=\frac{(m+1) A_{p h}}{2 \pi d^{n}} P_{T}
$$


TABLE II

EXPERIMENTAL PARAMETERS

\begin{tabular}{ll}
\hline Parameter & Value \\
\hline Transmission data Rate & $9.5 \mathrm{kbps}$ \\
Transmission baud Rate & $19 \mathrm{kbps}$ \\
Link range & 30 meter \\
LED color & Red \\
LED wavelength & $635 \mathrm{~nm}$ \\
Modulation type & OOK \\
Line coding & Manchester \\
LED power dissipation & 3 Watt \\
Number of LED array & 6 \\
LED lens viewing angle & $5^{\circ}$ \\
PD active area & $100 \mathrm{~mm}{ }^{2}$ \\
Transmitter \& Receiver height & $85 \mathrm{~cm}$ \\
Receiver sensitivity & $2.1 \mathrm{~V}$ \\
Gain levels & $30,40,50 \mathrm{~dB}$ \\
Mean Temperature & $13^{\circ} \mathrm{C}$ \\
Wind Speed & $11 \mathrm{~km} / \mathrm{hour}$ \\
Visibility & $12.0 \mathrm{kilometers}$ \\
Sea Level Pressure & $1025.11 \mathrm{hPa}$ \\
Average Humidity & 90 \\
\hline
\end{tabular}

Based on the fact that all the influencing parameters except the direct distance between transmitter and receiver were fixed. One can encapsulate the constant parameters over the experiment steps $\frac{(m+1) A_{p h}}{2 \pi} P_{T}$ in the term $A_{0}$, where a linear least-square relationship of the received power is expected over the variation in $d$ as the following:

$$
P_{R}=\frac{A_{0}}{d^{n}}
$$

To estimate the values of the two unknowns $A_{0}$ and $n$, we applied a regression process to the acquired received power as shown in Fig 6 . The results indicate that there is perfect agreement between the theoretical lambertian pathloss model expressed by (2) and the empirical measurements, where the estimated path-loss exponent $n \approx 2$.

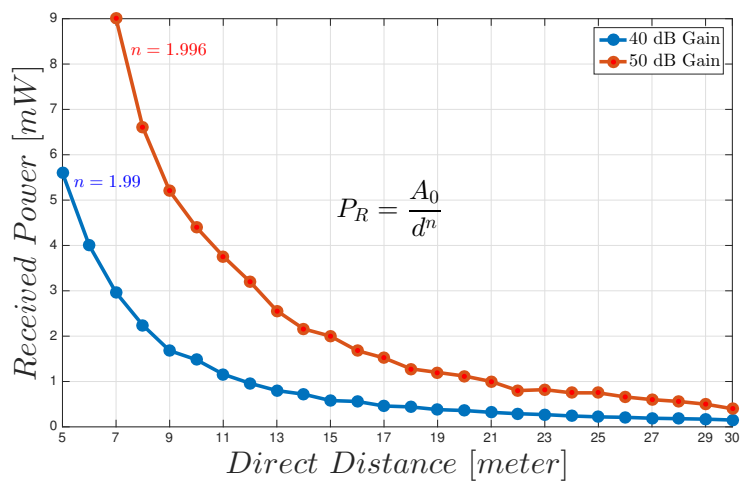

Fig. 6. Empirical estimation of the VLC link Path-loss exponent, $A_{50 d B}=$ $432.0479, n_{50 d B}=1.996, A_{40 d B}=139.4479, n_{40 d B}=1.99$.

Acquired data for link range shorter than 3 meters has shown unstable behavior at the receiver end due to the current saturation in the PD. We found out that, to overcome this problem, the applied gain, and the decision circuit threshold level should be dynamically adjusted in relevance to the intervehicle distance $d$. Based on our findings, and for the exact experiment conditions, we suggest using gain levels of 10$20 \mathrm{~dB}$ for ranges less than 3 meter and higher levels for the longer communication ranges.

Figure 7 shows the packet delivery ratio (PDR) results with respect to the inter-vehicle distance $d$., while receiver gain was set to $40 \mathrm{~dB}$. The results show that $100 \%$ of PDR is achievable for up to 30 meters inter-vehicle distance, which is realistic covering range for platooning application.

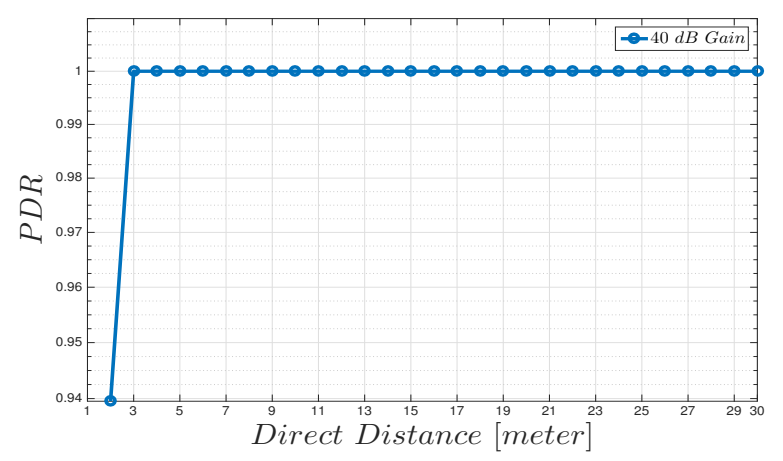

Fig. 7. Packet Delivery Ratio (PDR) of the VLC platform for $40 \mathrm{~dB}$ gain.

The link end-to-end delay performance is shown in Fig. 8. the acquired results show that the system can deliver the packets without exceeding $36 \mathrm{~ms}$ of delay time, which perfectly satisfies the requirements of the many hard safety applications, including the platoon.

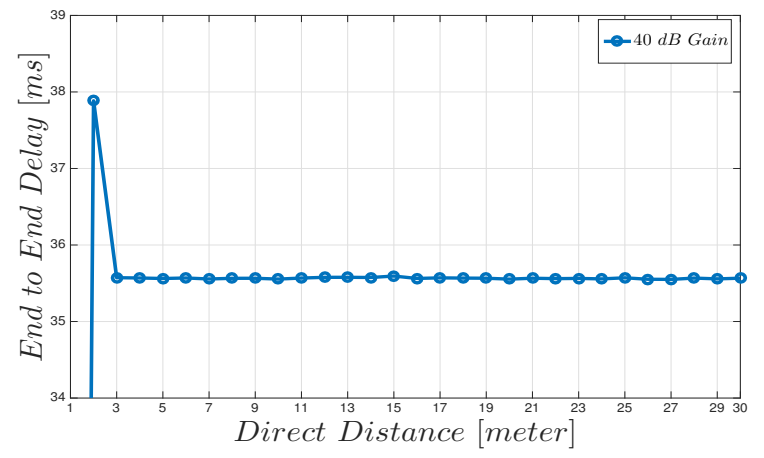

Fig. 8. The end-to-End delay performance of the VLC platform for $40 \mathrm{~dB}$ gain.

Figure 9 represents the throughput performance of the same platform. Here, throughput is the number of successfully received useful information bits during one second, excluding the headers, training bits, and the preambles.

One may think that the obtained throughput of $3.8 \mathrm{Kbps}$ is very low, especially if compared to e.g., $6 \mathrm{Mbps}$ of the IEEE 802.11p technology. However, it should be noted that there is no direct comparison in this context, the radio channel of the IEEE $802.11 \mathrm{p}$ is shared medium over approximately $1 \mathrm{~km}$ of geographical area, which may introduce hundreds of vehicles sharing the same channel. On the other hand, our proposed VLC channel for platoon is hardly shared by two transmitting vehicles, due to the strict LOS and FOV requirements. 


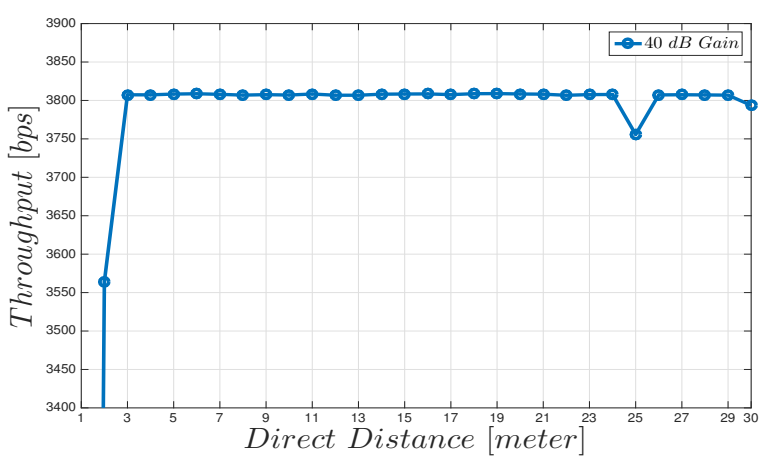

Fig. 9. The throughput performance of the VLC platform for $40 \mathrm{~dB}$ gain.

Moreover, our system throughput can be further improved, since the main bottleneck of the current system is due to the processing speed, platform implementation, and decision circuit, but not the physical VLC channel itself.

\section{Performance eValuation of VLC-Based PLATOON}

Platooning control is traditionally based on perception techniques that use vehicle embedded sensors, such as radars. More specifically, they usually use sensors to measure the distance between vehicles and apply, e.g., a classical proportional integral controller to control the velocity $v_{f}(t)$ with respect to time $t$ of the follower vehicle. For a measured inter-distance $d(t)$ at a time $t$, the velocity of the follower vehicle is given by:

$$
v_{f}(t)=K_{p} \cdot e(t)+K_{i} \cdot \int_{0}^{t} e(\tau) \mathrm{d} \tau,
$$

where $K_{p}$ and $K_{i}$ are respectively the proportional and the integral gains, the error $e(t)$ is

$$
e(t)=d_{r e f}-d(t) .
$$

These types of perception-based platooning control, however, suffer from the chain instability problem. The problem can be reduced or even eliminated if vehicles in the platoon can exchange information with the platoon leader. For this reason, a large number of efforts have been made on Cooperative Adaptive Cruise Control (CACC). For example, in the platooning controller designed in [16], the platoon members receive information from the vehicle in front as well as from the platoon leader.

The main advantage of this platooning control is that the system can be proven to be stable under a constant spacing policy. As illustrated by (7) (the parameters of are described in Table III), in order to ensure both the leader-follower and the predecessor-follower string stabilities, vehicles must compute their desired acceleration $\ddot{x}_{d e s}$ in parallel and in real time. To do so, each vehicle must receive the acceleration from their immediate predecessor $\ddot{x}_{i-1}$, and the leader vehicle's speed $V_{l}$, where platoon leader acceleration $\ddot{x}_{l}$ must be available to all platoon members [17]. Apparently, the VLC technology can be used for the former type of information exchange, i.e., bumper-to-bumper communication. On the other hand, for the latter type of information exchange, i.e., communication from the leader to the platoon members, multi-hop data delivery based on VLC is maybe an approach, but it is probably more logic to consider the radio communication technology.

TABLE III

TERMS DESCRIPTION OF CONSTANT SPACING PLATOON CONTROL MODEL

\begin{tabular}{ll}
\hline Symbol & Description \\
\hline$\ddot{x}_{\text {des }}$ & ith vehicle desired acceleration \\
$\ddot{x}_{i-1}$ & Acceleration of the vehicle preceding the $i t h$ vehicle \\
$\ddot{x}_{l}$ & Platoon leader acceleration \\
$V_{i}$ & ith vehicle velocity \\
$V_{l}$ & Platoon leader velocity \\
$C_{1}$ & Control gain ( values $0<C 1<1)$ \\
$\omega_{n}$ & Controller bandwidth \\
$\varepsilon_{i}$ & Spacing error between Vehicles \\
$\xi$ & Damping ratio (typically equal to 1 for critical damping) \\
\hline
\end{tabular}

$$
\begin{aligned}
\ddot{x}_{d e s}= & \left(1-C_{1}\right) \ddot{x}_{i-1}+C_{1} \ddot{x}_{l} \\
& -\left(2 \xi-C_{1}\left(\xi+\sqrt{\xi^{2}-1}\right)\right) \omega_{n} \dot{\varepsilon}_{i} \\
& -\left(\xi+\sqrt{\xi^{2}-1}\right) \omega_{n} C_{1}\left(V_{i}-V_{l}\right)-\omega^{2}{ }_{n} \varepsilon_{i} .
\end{aligned}
$$

In a simpler model, the individual platoon members can require information only from their preceding vehicles, i.e., bumper-to-bumper communication. This type of platooning control shows inferior stability performance compared to that presented in [16]. Nevertheless, the performance should be much better compared to the classical perception-based (which uses only the vehicle local sensors) technology. Since the scope of this paper is VLC, which perfectly fits to bumper-to-bumper communication, we apply our VLC platform to this type of platooning control (requires only bumper-to-bumper communication) and evaluate its performance.

Assuming that a platoon consists of homogeneous autonomous vehicles, we evaluate the performance of platooning control, where individual vehicles use the reference speed of their preceding vehicles. In the evaluations, the Inria's autonomous vehicles, Cycab [18], are modeled in the Simulink, to which our VLC platform is connected by a serial port to realize the bumper-to-bumper communication (see Fig 10,

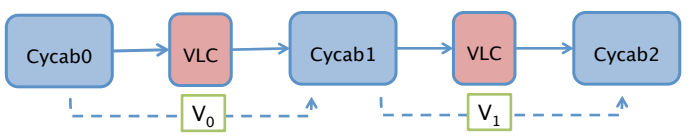

Fig. 10. Simulink Model for 3 Cycabs forming VLC-based platoon

The performance of the VLC-based platoon is compared to that of perception-based platoon, which does not use communication. The results of the perception-based and VLC-based platoons are depicted in Figs. 11 and 12, respectively, which plot the velocities of the individual vehicles with respect to the simulation time. As Fig. 11 shows, in the perception-based platoon, each follower vehicle needs 
approximately 1 second to adjust its speed to that of the preceding vehicle. In contrast, Fig. 12 shows that when our VLC system is used to allow inter-vehicle communication, the time necessary to adjust the velocity is only $35 \mathrm{~ms}$, clearly indicating the benefits of using the VLC system for intra-platoon communication.

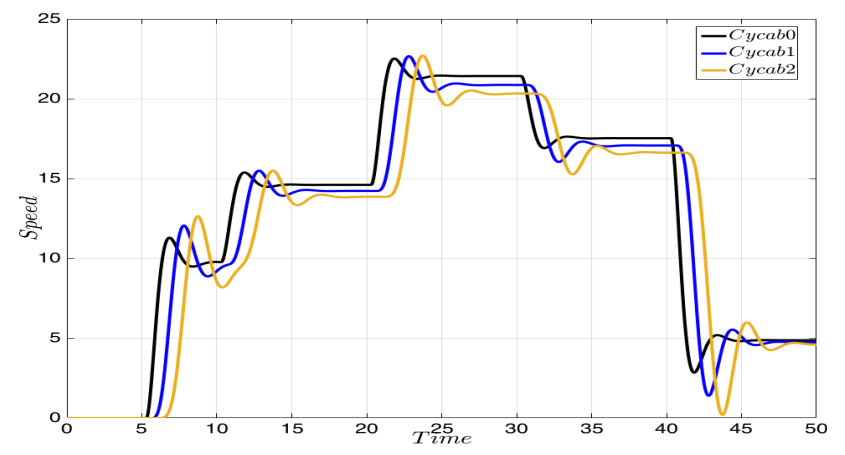

Fig. 11. Performance of the perception-based platoon.

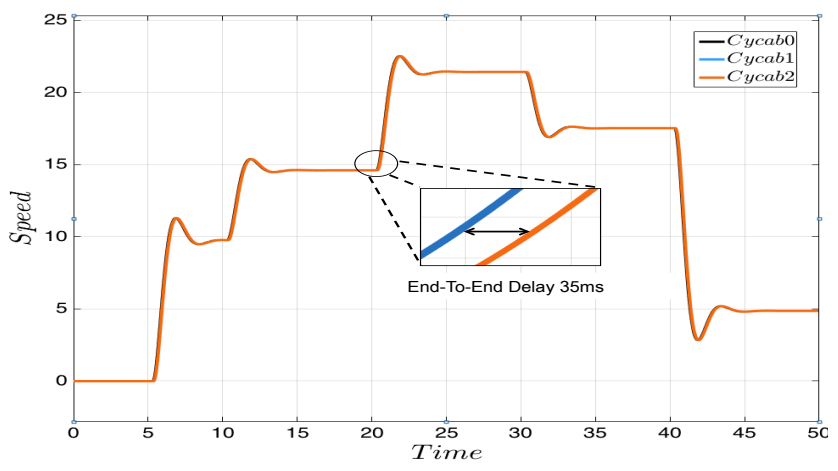

Fig. 12. Performance of the VLC-based platoon.

\section{CONCLUSIONS}

Visible light communications have been lately considered as a supportive candidate for vehicular communications, especially in high dense traffic scenarios. However, very few effort have been made to study and address the technology for specific ITS applications considering the real-world conditions. In this work, we tried to fill the missing gap. We presented the system architecture, implementation, and the evaluation of a practical outdoor VLC platform for autonomous platoons. We performed several tests that intended to measure the performances of the received optical power, packets delivery ratio, link throughput and the end-to-end delay. The measured VLC path-loss characteristics showed a substantial agreement with the theoretical model. The platform operated with an excellent end-to-end communication performances regarding the platoon safety requirements of both PDR and delay. We also evaluated the impact of the actual VLC system on the platoon performance using a Cycab Simulink model and compared the results to those of perception-based platooning control. In our ongoing work, and after integrating the platform with an actual Cycab, we aim to improve the system bit rate and re-perform the experiment under the presence of more challenging weather conditions; such as fog, rain, and snow. A sufficient handover algorithm between VLC and radio communication and/or laser sensing systems is intended to be implemented on a future work basis.

\section{ACKNOWLEDGMENT}

The authors would like to acknowledge the support of all RITS team members. Also, grateful to Vicente MILANES for providing the Cycab Simulink model.

\section{REFERENCES}

[1] IEEE Std 802.15.7-2011, pp. 1-309, Sept 2011.

[2] S. Eichler, "Performance evaluation of the ieee $802.11 \mathrm{p}$ wave communication standard," in Vehicular Technology Conference, 2007. VTC2007 Fall. 2007 IEEE 66th, Sept 2007, pp. 2199-2203.

[3] P. Varaiya, "Smart cars on smart roads: problems of control," Automatic Control, IEEE Transactions on, vol. 38, no. 2, pp. 195-207, Feb 1993.

[4] "Vehicle Dynamics and Control | Rajesh Rajamani | Springer," 2006.

[5] P. Fernandes and U. Nunes, "Platooning of autonomous vehicles with intervehicle communications in sumo traffic simulator," in Intelligent Transportation Systems (ITSC), 2010 13th International IEEE Conference on, Sept 2010, pp. 1313-1318.

[6] S. Biswas, R. Tatchikou, and F. Dion, "Vehicle-to-vehicle wireless communication protocols for enhancing highway traffic safety," Communications Magazine, IEEE, vol. 44, no. 1, pp. 74-82, Jan 2006.

[7] P. Fernandes and U. Nunes, "Platooning with ivc-enabled autonomous vehicles: Strategies to mitigate communication delays, improve safety and traffic flow," Intelligent Transportation Systems, IEEE Transactions on, vol. 13, no. 1, pp. 91-106, March 2012.

[8] J. Kahn and J. Barry, "Wireless infrared communications," vol. 85, no. 2, pp. $265-298$, Feb. 1997.

[9] J. Peck, G. Ashburner, and M. Schratz, "Solid state led lighting technology for hazardous environments; lowering total cost of ownership while improving safety, quality of light and reliability," in Petroleum and Chemical Industry Conference Europe Conference Proceedings (PCIC EUROPE), 2011, June 2011, pp. 1-8.

[10] Y. H. Kim, W. Cahyadi, and Y. H. Chung, "Experimental demonstration of vlc-based vehicle-to-vehicle communications under fog conditions," Photonics Journal, IEEE, vol. 7, no. 6, pp. 1-9, Dec 2015.

[11] Y.-H. Kim, W. A. Cahyadi, and Y. H. Chung, "Experimental demonstration of led-based vehicle to vehicle communication under atmospheric turbulence," in Information and Communication Technology Convergence (ICTC), 2015 International Conference on, Oct 2015, pp. 1143-1145.

[12] M. Abualhoul, M. Marouf, O. Shagdar, and F. Nashashibi, "Platooning control using visible light communications: A feasibility study," in Intelligent Transportation Systems - (ITSC), 2013 16th International IEEE Conference on, Oct 2013, pp. 1535-1540.

[13] U. Department of Transportation, "Vehicle safety communications project task 3." http://www.ntis.gov/

[14] intempora, "Rtmaps 4," https://intempora.com

[15] M. Abualhoul, M. Marouf, O. Shag, and F. Nashashibi, "Enhancing the field of view limitation of visible light communication-based platoon," in Wireless Vehicular Communications (WiVeC), 2014 IEEE 6th International Symposium on, Sept 2014, pp. 1-5.

[16] R. Rajamani, H.-S. Tan, B. K. Law, and W.-B. Zhang, "Demonstration of integrated longitudinal and lateral control for the operation of automated vehicles in platoons," Control Systems Technology, IEEE Transactions on, vol. 8, no. 4, pp. 695-708, Jul 2000.

[17] W. Dunbar and D. Caveney, "Distributed receding horizon control of vehicle platoons: Stability and string stability," Automatic Control, IEEE Transactions on, vol. 57, no. 3, pp. 620-633, March 2012.

[18] INRIA, "Cycab autonomous vehicle," http://www.inria.fr/en/news/ news-from-inria/cycab 